\title{
Prevalence of Anemia and Its Associated Factors in Antiretroviral-Treated HIV/AIDS-Positive Adults from 2013 to 2018 at Debre Berhan Referral Hospital, Ethiopia
}

\author{
Yared Asmare Aynalem $\mathbb{D}^{1},{ }^{1}$ Wondimeneh Shibabaw Shiferaw, ${ }^{1}$ and Zeleke Woldiye ${ }^{2}$ \\ ${ }^{1}$ College of Health Science, Debre Berhan University, Debre Berhan, Ethiopia \\ ${ }^{2}$ Debre Berhan Referral Hospital, Debre Berhan, Ethiopia \\ Correspondence should be addressed to Yared Asmare Aynalem; yaredasmare123@gmail.com
}

Received 6 August 2019; Revised 9 February 2020; Accepted 17 February 2020; Published 11 March 2020

Academic Editor: Myriam Labopin

Copyright (C) 2020 Yared Asmare Aynalem et al. This is an open access article distributed under the Creative Commons Attribution License, which permits unrestricted use, distribution, and reproduction in any medium, provided the original work is properly cited.

\begin{abstract}
Introduction. Anemia was defined as a hemoglobin level of less than or equal to $13.9 \mathrm{~g} / \mathrm{dl}$ for male and less than or equal to $12.2 \mathrm{~g} / \mathrm{dl}$ for female adults. It is one of the most common hematological abnormalities in people living with human immunodeficiency virus/acquired immunodeficiency syndrome (HIV/AIDS) and is a determining factor for disease progression and death. Among the countries in sub-Saharan Africa, Ethiopia is one of the most affected nations by HIV. Therefore, this study aimed to assess the prevalence of anemia and its associated factors among HIV-positive adults that had received antiretroviral treatment (ART) at Debre Berhan Referral Hospital. Methods. An institution-based, descriptive, cross-sectional study was conducted involving 263 adults with HIV/AIDS that had undergone ART at Debre Berhan Referral Hospital, Ethiopia. Data were collected from patient charts using systematic sampling with a pretested data extraction tool and entered using EpiData 3.1. Variables having a $p$ value $\leq 0.25$ in the bivariate were fitted to a multivariable regression model with a $95 \%$ confidence interval. $p$ values $\leq 0.05$ were considered statistically significant in the multivariate analysis. Results. Among the $263 \mathrm{HIV}$-positive patients, 237 (90.11\%) were included in the final analysis. The overall prevalence of anemia was $26.2 \%$. Factors that were significantly associated with anemia were past opportunistic infections, patients being in WHO clinical stage III and IV, and a BMI $<18.5$. Conversely, those patients who took anti-TB medication were less likely to have anemia. Conclusion. Our study shows that the severity of anemia among HIV/AIDS patients that had undergone ART is lower than most studies conducted in Ethiopia. We also found that opportunistic infection, WHO clinical staging, anti-TB treatment, and low BMI were significantly associated with anemia. Therefore, routine screening of patient nutritional status and opportunistic infections may be useful in predicting and controlling anemia in HIV/ AIDS patients.
\end{abstract}

\section{Introduction}

According to the World Health Organization (WHO), anemia is a condition in which the number or size of red blood cells and, thus, hemoglobin (Hgb) concentration fall below the required level to transport oxygen effectively. It is also defined as a condition in which the Hgb content of the blood is lower than normal for a person's age, gender, and environment, resulting in the reduction of oxygen-carrying capacity of the blood. It affects more than 2 billion people globally, which is $\sim 30 \%$ of the global population. Moreover, anemia is of particular concern for developing countries, where its effects extend beyond human health and significantly impact the social and economic welfare of the nation $[1,2]$. It might have a negative effect on quality of life, mortality, morbidity, and socioeconomic progress of a country at large. It affects more developing countries than the developed countries [2]. Based on recent estimates from the World Health Organization (WHO), the prevalence of anemia is $24.8 \%$ globally [3]

Anemia is one of the most frequently reported hematological complications in people afflicted with human 
immunodeficiency virus/acquired deficiency syndrome (HIV/AIDS). Although not well studied, it is estimated that about $60-80 \%$ of late-stage HIV patients are anemic. In these patients, $22 \%$ of anemia is thought to be caused by HIV treatments including antiretroviral medications $[4,5]$. Moreover, anemia is considered one of the key independent predictors of HIV disease progression [6-9]. Even if anemia responds to antiretroviral therapy, it often persists in many patients despite therapy, and such persistent anemia continues to negatively affect prognosis regardless of drug response [10]. Additionally, in asymptomatic HIV/AIDS patients, it is estimated that up to $30 \%$ are anemic.

The cause of anemia is multifactorial. For instance, females are more likely to be anemic compared with males worldwide. In a study conducted in the US, it was reported that women had a $71 \%$ greater prevalence of anemia than men [4]. Additional contributors to an anemic state include nutritional deficiencies, opportunistic, and parasitic infections, as well as the presence of a chronic infection, which may decrease erythropoiesis [11]. Some of the factors associated with anemia in HIV/ AIDS patients in Ethiopia are a low CD4 count, WHO clinical stage III and IV, opportunistic infections (OIs), and a low BMI [12-15].

Ethiopia is ranked as one of the countries with the highest HIV burden worldwide, with about 1.1 million people living with HIV and about 14,405 new HIV infections being reported in 2016 [16]; anemia was a major comorbidity in many of these cases $[13,14,17]$. In Ethiopia, despite the availability of HAART for more than 13 years, there is still substandard data on the prevalence of anemia and its associated factors in HIV-positive adults. Therefore, this study aims to assess the prevalence of anemia and its associated factors among HIV-positive patients that have received antiretroviral treatment (ART) at Debre Berhan Referral Hospital (DBRH), Ethiopia, from 2013 to 2018.

\section{Materials and Methods}

2.1. Study Design, Area, Period, and Populations. This study was an institution-based, descriptive, cross-sectional study involving HIV-positive adults that had received ART from April to March 2019. The study was conducted in DBRH, North Shewa Zone, Amhara, Ethiopia. Based on the hospital medical record report, the total number of HIV-positive individuals that had received ART from 2013 to 2018 was 847 (285 males and 562 females). All HIV-positive individuals who had received ART and were aged 18 years or older were our source population, and all HIV-positive individuals who had received ART, were aged 18 years or older, and had followed up at DBRH from 2013 to 2018 were our study population.

2.2. Eligibility Criteria. Individuals were considered eligible if they were aged 18 years or older, had a confirmed HIV infection upon follow-up at DBRH, and had a detailed history and laboratory results such as Hgb and CD4 counts.
Those individuals that did not have a detailed history and laboratory results and patients with incomplete records were excluded.

2.3. Sample Size Determination and Sampling Technique and Procedure. The sample size was calculated using the single population proportion sampling formula with the following assumptions: $5 \%$ marginal error, $\mathrm{Z}$ is the value corresponding to the $95 \%$ confidence interval $(\mathrm{CI})=1.96$, and proportion of anemia $(P)=53.6 \%$ [18], which was taken from a study at Arba Minch, Ethiopia. Using these parameters, the sample size was found to be 382 . Because the source population was less than 10,000 , we used the correction formula to determine the final sample size of 263. Patient chart numbers were taken from the HIV/AIDS registration book. The systematic random sampling technique was employed to identify the study units using the ART unique numbers from the registration books.

\subsection{Study Variable}

2.4.1. Dependent Variable. Prevalence of anemia in HIVpositive adults that had received ART.

\subsubsection{Independent Variables}

(1) Sociodemographic Characteristics. Age, sex, marital status and occupation of mother or caregiver, residence, parental status (alive or dead), and family size.

(2) Baseline Clinical and Laboratory Information. CD4 count, Hgb, past opportunistic illnesses, tuberculosis (TB) test, WHO staging, BMI, developmental or functional history, and treatment.

(3) ART and Other Medication Information. Regimen at follow-up, adherence to ART, cotrimoxazole preventive therapy, side effects of ART drugs, regimen change, and regimen stopped.

2.5. Data Collection Methods and Tools. The data tool was developed from the standardized ART entry and follow-up form currently used by ART clinics. It was also developed by referring to the relevant literature. The available information on the patient records was first observed, and an appropriate data extraction format was prepared in English and then was translated into Amharic. The data were then collected by the principal investigators using the prepared data collection format on the already existing records. Analysis of serum $\mathrm{Hb}$ levels was a routine laboratory investigation. Therefore, it was collected from the laboratory results done and documented at the time of data collection or done recently, before two weeks back.

2.6. Data Quality Assurance. Data quality was assured by designing a proper data collection checklist. The checklist was evaluated by experienced researchers. The data 
collection instrument was tested on $5 \%$ of the sample size. Language clarity, appropriateness of data collection tools, estimate of the time required, and the necessary amendments were considered based on the test. During data collection, close supervision and monitoring was carried out by supervisors and the investigator to ensure the quality of the data. Finally, all of the collected data were checked by the supervisor and the investigator for its completeness and consistency during data management, storage, and analysis. Consistency was examined through random selection of checklist. Accuracy of the data was also assured by everyday on-site cross-checks during data collection.

2.7. Data Processing and Analysis. Data were cleaned, edited, and coded. Any errors were identified and corrected after review of the original data using the code numbers. The data were then entered using EpiData version 3.1 and analyzed using SPSS 24 statistical software. Anemia was defined based on hematological reference values for the adult Ethiopian population [19]. Consequently, it was defined as an $\mathrm{Hgb}$ $\leq 13.9 \mathrm{~g} / \mathrm{dl}$ for males and $\leq 12.2 \mathrm{~g} / \mathrm{dl}$ for females. It was also categorized into mild $(10-12.2 \mathrm{~g} / \mathrm{dl}$ for females and $10-13.9 \mathrm{~g} / \mathrm{dl}$ for males), moderate $(8-10 \mathrm{~g} / \mathrm{dl})$, and severe $(<8 \mathrm{~g} / \mathrm{dl})$ for both sexes. Descriptive statistics were used to present the sociodemographic and other anemia-related factors. In the ultimate multivariable models, the level of multicollinearity was checked and fitted using variance inflation factor and tolerance and found within a tolerable range (all variables of the variance inflation factor value were $<3.2$ ) and tolerance (all variable values $>0.2$ ). The binary regression model assumption was checked using a goodness test such as the Hosmer-Lemeshow goodness of fit, and variables having $p$ values $>0.05$ were considered as fulfilling the assumption. The bivariate regression model was fitted for each explanatory variable. Accordingly, those variables having $p$ values $\leq 0.25$ in the bivariate analysis were fitted to the multivariable regression model with $95 \%$ CI. $p$ values $\leq 0.05$ were considered statistically significant in the multivariate analysis.

\section{Results}

3.1. Sociodemographic Characteristics of the Study Participants. Of the $263 \mathrm{HIV}$-positive adult patient records reviewed, 237 (90.11\%) were included in the final analysis. Around two-thirds (67.9\%) of these participants were females, and the majority of them $(83.5 \%)$ came from an urban area. Half of the study participants were married. The mean age of the study participants was 35 years old, and the minimum and maximum ages were 18 and 71 years old, respectively. Among the eligible patients, 92 (38.8\%) were 15-30 years old, $110(46.45 \%)$ were $31-45$ years old, and 35 $(14.8 \%)$ were older than 45 years old (Table 1$)$.

3.2. Baseline Clinical, Laboratory, and ART Information. Among the 237 participants, 88 (37.1\%) had past opportunistic infections. Of these individuals, 25 (28.4\%) had
TABLE 1: Sociodemographic characteristics of patients.

\begin{tabular}{lccc}
\hline Characteristics & & Frequency & Percentage \\
\hline \multirow{2}{*}{ Sex } & Male & 76 & 32.1 \\
& Female & 161 & 67.9 \\
Age & $18-30$ & 92 & 38.8 \\
& $31-45$ & 110 & 46.4 \\
Residence & $>45$ & 35 & 14.8 \\
& Urban & 198 & 83.5 \\
\multirow{4}{*}{ Marital status } & Rural & 39 & 16.5 \\
& Single & 29 & 12.2 \\
& Married & 123 & 51.9 \\
& Divorced & 56 & 23.6 \\
& Widowed & 29 & 12.2 \\
\hline
\end{tabular}

candidiasis, 18 (20.5\%) had pneumonia, and $16(18.2 \%)$ had herpes zoster. Additionally, the majority of the patients (227 $(95.8 \%))$ were functional and working and more than half of the participants were at WHO clinical stage I. More than half of the study participants also had a BMI $<18.5 \mathrm{~kg} / \mathrm{m}^{2}$ (Table 2).

\subsection{RT Treatment and Other Medication-Related Charac-} teristics of the Participants. Only $45.3 \%$ of the participants had a history of regimen change throughout the entire follow-up. From those who had a history of regimen change, toxicity or drug side effects (54.8\%) is the most common reason for the alteration, with drugs being out of stock (24.6\%) and clinical failure (9.8\%) also being prevalent within the group. In addition, among those who had side effects from the drug (17.84\%) during follow-up, anemia (30.8\%) was found to be the most common complaint followed by nausea (20.8\%) and rash (12.3\%). Almost all study participants received cotrimoxazole prophylaxis (223 (94.1\%)), and zidovudine-lamivudine-efavirenz (AZT-3TCEFV) was given to $228(96.2 \%)$ of the patients at follow-up (Table 3).

3.4. Prevalence of Anemia among HIV-Positive Patients. The prevalence of anemia among HIV-positive adults on HAART was $26.2 \%(62 / 237)$. Of those, $47 \%$ and $39 \%$ had mild and moderate anemia, respectively (Figure 1).

\subsection{Anemia-Associated Factors among HIV-Positive Patients} on HAART. Both the bivariate and multivariate logistic regressions were done to assess the anemia-associated factors among HIV-positive patients. Our bivariate analysis showed that factors including past OIs, WHO clinical staging (stage III and IV), BMI, previous anti-TB treatment, and OI during follow-up were associated with anemia. Moreover, in our multivariate logistics analysis model, only having a past OI, WHO clinical staging (stage III and IV), previous anti-TB treatment, and OI were associated with anemia. Multivariate analysis revealed that patients who had an OI were 1.6 times more likely to develop anemia compared with those who did not have an OI (AOR: 1.6 (95\% CI: 1.59-4.33)). Patients at WHO clinical stage III and IV were almost three times more likely to die compared with those at 
TABLE 2: Baseline clinical and laboratory information of patients.

\begin{tabular}{|c|c|c|c|}
\hline Characteristics & & Frequency & Percentage \\
\hline \multirow{7}{*}{ Past opportunistic infection } & Herpes zoster & 16 & 18.2 \\
\hline & Candidiasis (oral and esophageal) & 25 & 28.4 \\
\hline & Pneumocystis pneumonia & 18 & 20.5 \\
\hline & Past TB test $\quad$ Positive & 27 & 11.4 \\
\hline & Negative & 210 & 88.6 \\
\hline & Diarrhea & 11 & 12.5 \\
\hline & Others & 18 & 20.3 \\
\hline \multirow{3}{*}{ Functional status } & Working & 227 & 95.8 \\
\hline & Ambulatory & 10 & - \\
\hline & Stage I & 146 & 61.6 \\
\hline \multirow[t]{2}{*}{ WHO clinical staging } & Stage II & 43 & 18.1 \\
\hline & Stage III and IV & 48 & 20.3 \\
\hline \multirow{3}{*}{ CD4 counts } & $<350$ & 74 & 31.2 \\
\hline & $\geq 350$ & 163 & 68.8 \\
\hline & $<18.5$ & 66 & 27.8 \\
\hline \multirow[t]{2}{*}{ BMI $\left(\mathrm{kg} / \mathrm{m}^{2}\right)$} & $18.5-24.9$ & 123 & 51.9 \\
\hline & $\geq 25.0$ & 48 & 20.3 \\
\hline
\end{tabular}

TABLE 3: ART treatment and other medication-related characteristics of patients.

\begin{tabular}{lccc}
\hline Characteristics & & Frequency & Percentage \\
\hline & CD4 $<350$ and WHO stage IV & 16 & 6.8 \\
ART eligibility criteria & WHO stage II and III & 26 & 195 \\
& Without criteria & 225 & 82.3 \\
OI prophylaxis & Yes & 12 & 130 \\
& No & 107 & -9.9 \\
ART side effects during follow-up & Yes & 103 & 44.8 \\
& No & 134 & 43.3 \\
Regimen change during follow-up & Yes & 56.7 \\
\hline
\end{tabular}

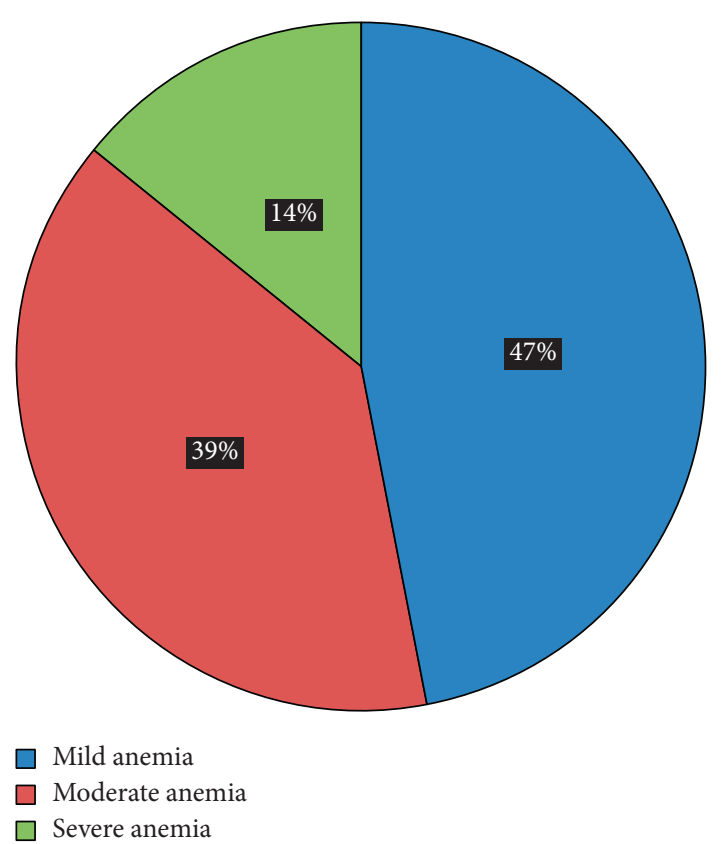

FIGURE 1: Hgb classification of the participants from the DBRH ART clinic who were on HAART.

WHO clinical stage I and II (AOR: 2.98 (95\% CI: 1.25-7.12)). In this study, patients who had taken anti-TB medication were $80 \%$ less likely to have anemia than those patients who did not take anti-TB medication (AOR: 0.2 (95\% CI: 0.12-0.91)). Moreover, HIV-positive patients who had a $\mathrm{BMI} \leq 18$ were three times more likely to have anemia than those individuals having a $\mathrm{BMI} \geq 25$ (AOR: 3.1 (95\% CI: 1.33-7.26)) (Table 4).

\section{Discussion}

Anemia is a major concern for those individuals who are suffering from HIV/AIDS as it is a significant determinant of disease progression. This study showed that $26.2 \%$ of $\mathrm{HIV}$-positive adults that were treated at DBRH from 2013 to 2018 had anemia. This is consistent with the results from studies in Rwanda (29\%) [20], Nigeria (24.3\%) [6], and South Africa (25.8\%) [21]. However, the prevalence of anemia in HIV-positive patients was lower in this study than in studies conducted in other areas of Ethiopia including Debre Tabor (34\%) [15], Arba Minch (53.6\%) [18], Gondar (35\%) [7], and Zewditu Memorial Hospital (42.9\%) [14]. Similarly, our results were much lower than those found in studies of patients from Nigeria (60.61\%) [22], Tanzania (56\%) [23], Bayamón, Puerto Rico (41\%) [24], Nepal (55.8\%) [25], and China (39.2\%) [26]. However, the overall prevalence of anemia among HIV-positive adults was higher than that found in patients from Jimma $(23.1 \%)$ [8], India (16.2\%) [9], and Ghana (23.8\%) [27]. In this study, the prevalence of mild, moderate, and severe anemia 
TABLE 4: Bivariate and multivariate logistics regression analysis of variables.

\begin{tabular}{|c|c|c|c|c|c|}
\hline \multirow{2}{*}{ Variables } & & \multicolumn{2}{|c|}{ Anemia } & \multirow{2}{*}{ COR (95\% CI) } & \multirow{2}{*}{$\operatorname{AOR}(95 \% \mathrm{CI})$} \\
\hline & & Non-anemic & Anemic & & \\
\hline \multirow{2}{*}{ Sex } & Male & 21 & 55 & Reference & \\
\hline & Female & 42 & 119 & $2.1(0.84-3.68)$ & $1.16(0.23-2.50)$ \\
\hline \multirow{2}{*}{ OIs } & Yes & 37 & 50 & $0.3(0.15-0.51)^{*}$ & $1.6(1.59-4.33)^{*}$ \\
\hline & No & 26 & 124 & Reference & \\
\hline \multirow{2}{*}{ WHO staging } & Stage I and II & 36 & 12 & Reference & \\
\hline & Stage III and IV & 27 & 162 & $0.5(0.2-0.89)^{*}$ & $2.9(1.25-7.12)^{*}$ \\
\hline \multirow{2}{*}{ Anti-TB Rx } & Yes & 25 & 24 & Reference & \\
\hline & No & 38 & 150 & $0.4(0.1-1.11)^{*}$ & $0.2(0.12-0.91)^{*}$ \\
\hline \multirow{3}{*}{ BMI } & $<18$ & 24 & 42 & $3.24(1.63-6.45)$ & $3.1(1.33-7.26)^{*}$ \\
\hline & $18.5-24.9$ & 22 & 101 & $1.2(0.42-3.03)^{*}$ & $1.3(0.43-3.73)$ \\
\hline & $\geq 25$ & 17 & 31 & Reference & \\
\hline
\end{tabular}

* Indicates significance at a $p$ value $<0.05$.

was $47 \%, 39 \%$, and $14 \%$, respectively, which is lower than the results presented from a study in China [26]. These differences in the burden of anemia among HIV-positive patients may be related to differences in the sample size (e.g., sample size was large in Nigeria), sociodemographic disparity, characteristics of the study participants, and changes in treatment modalities. Additionally, all participants in this study were HIV-positive patients on ART. This might also indirectly show the efficacy of HAART in reducing HIV-associated anemia by reducing the incidence of OIs, chronic illnesses, and improving the nutritional status of patients.

In this study, OI, WHO clinical staging, low BMI, and anti-TB treatment were significantly associated with anemia. Indeed, advanced WHO clinical staging (III and IV) was found to be an independent associated factor of anemia for patients with HIV/AIDS. Adults with advanced WHO clinical stage (III and IV) HIV/AIDS at the time of ART initiation were almost three times less likely to have anemia compared with those in WHO clinical disease stage I and II at the time of ART initiation. This is supported by a previous study conducted in Ethiopia [28].

In this study, the odds of getting anemia among HIVpositive patients that had a BMI $\leq 18$ were three times higher than those individuals having a BMI $\geq 25$. This is in line with a study from Rwanda [29]. The association between anemia and low BMI may stem from the fact that HIV/AIDS causes malnutrition in many individuals due to reduced appetite, OIs, and treatment side effects. This might lead to difficulty in ingestion and diarrhea, which might limit nutrient uptake including iron.

This study also noted that the likelihood of having anemia was higher among patients with OIs. HIV-positive adults with OIs were 1.6 times more likely to have anemia compared with patients with no OI. This is supported by a previous study done in Jimma hospital [8]. This could be due the fact that even if ART is initiated for all HIV-positive patients as early as possible, most patients present for care and treatment at late clinical stages. Therefore, they might be diagnosed as having OI, which are the major causes of morbidity and mortality including anemia among HIVinfected patients.
Another anemia-associated factor for HIV-positive patients found in this study was anti-TB treatment. Adults taking an anti-TB treatment at the time of ART initiation were $80 \%$ less likely to have anemia compared with those who were not taking an anti-TB treatment. This is supported by a study done in Ethiopia and Tanzania [23, 30]. This association between anemia and anti-TB treatment may be due to the fact that TB is the most frequent life-threatening $\mathrm{OI}$ and leading cause of death among HIV-infected people. It increases HIV replication through the process of immune activation, which leads to an increased viral load. Thus, it is vital for both TB and HIV control programs to be synergistic to alleviate the double burden of TB/HIV in populations affected by both diseases. Moreover, TB management programs might be improved if they were able to reduce anemia in their coinfected patients.

\section{Conclusions}

This study showed that the prevalence of anemia in HIV/ AIDS patients is lower than that in most studies conducted in Ethiopia. We also found that the presence of OIs, WHO clinical stage III and IV, low BMI, and anti-TB treatment were significant factors associated with anemia in these patients. Therefore, the government needs to focus on repetitive screening of $\mathrm{HIV}$-infected patients with low $\mathrm{Hgb}$ levels and had opportunistic infection and strengthen appropriate treatment of $\mathrm{TB}$ patients and nutritional diversification.

\section{Abbreviations}

AIDS: Acquired immunodeficiency syndrome

AZT: Zidovudine

DBRH: Debre Berhan Referral Hospital

DBU: Debre Berhan University

HAART: Highly active antiretroviral therapy

Hgb: Hemoglobin

HIV: Human immunodeficiency virus

OIs: Opportunistic infections

PLWHA: People living with HIV/AIDS

SPSS: $\quad$ Statistical package for social science

WHO: World Health Organization. 


\section{Data Availability}

The data are accessible from the corresponding author upon reasonable request.

\section{Ethical Approval}

A written letter of permission from the research committee at Debre Berhan University, College of Health Science, was obtained and submitted to DBRH. Oral permission was obtained from DBRH coordinators. Confidentiality of the patient profiles was ensured throughout the research process.

\section{Conflicts of Interest}

The authors declare no conflicts of interest.

\section{Authors' Contributions}

All authors participated in the study design, interpretation of the data, and writing of the paper, and all authors have seen and approved the final version of the paper.

\section{Acknowledgments}

The authors would like to thank Debre Berhan University for the ethical clearance. They also acknowledge the study participants, data collectors, and the supervisor.

\section{References}

[1] WHO/CDC, Worldwide Prevalence of Anemia 1993-2005, WHO Global Database on Anemia, WHO/CDC, Atlanta, GA, USA, 2008.

[2] E. McLean, M. Cogswell, I. Egli, D. Wojdyla, and B. de Benoist, "Worldwide prevalence of anaemia, WHO vitamin and mineral nutrition information system, 1993-2005," Public Health Nutrition, vol. 12, no. 4, pp. 444-454, 2009.

[3] D. Benoist, E. McLean, I. Egli, and IM. Congswel, "Worldwide prevalence of anemia," WHO Global Database on Anemia,WHO, Geneva, Switzerland, 2015.

[4] A. M. Levine, K. Berhane, L. Masri-Lavine et al., "Prevalence and correlates of anemia in a large cohert of HIV -infected women: women's interagency HIV study," Journal of Acquired Immune Deficiency Syndromes, vol. 26, no. 1, pp. 2835, 1999.

[5] WHO/UNAIDS, "Global AIDS report 2011," WHO, Geneva, Switzerland, 2011.

[6] B. A. Denue, I. M. Kida, A. Hammagabdo, A. Dayar, and M. A. Sahabi, "Prevalence of anemia and immunological markers in HIV-infected patients on highly active antiretroviral therapy in Northeastern Nigeria," Infectious Diseases: Research and Treatment, vol. 6, pp. 25-33, 2013.

[7] G. Ferede and Y. Wondimeneh, "Prevalence and related factors of anemia in HAART-naive HIV positive patients at Gondar University Hospital, Northwest Ethiopia," BMC Hematology, vol. 13, no. 1, p. 8, 2013.

[8] L. Gedefaw, T. Yemane, Z. Sahlemariam, and D. Yilma, "Anemia and risk factors in HAART naive and HAART experienced HIV positive persons in south west Ethiopia: a comparative study," PLoS One, vol. 8, no. 8, Article ID e72202, 2013.

[9] M. B. Kulkarni, M. M. Bhalerao, S. U. Mungal, and S. P. Dube, "Anemia in people living with HIV/AIDS: a cross sectional study from India," IOSR Journal of Dental and Medical Sciences, vol. 14, no. 2, pp. 04-08, 2015.

[10] A. J. Redig and N. Berliner, "Pathogenesis and clinical implications of HIV-related anemia in 2013," Hematology, vol. 2013, no. 1, pp. 377-381, 2013.

[11] J. C. Calis, M. B. van Hensbroek, R. J. de Haan, P. Moons, B. J. Brabin, and I. Bates, "HIV-associated anemia in children: a systematic review from a global perspective," AIDS, vol. 22, no. 10, pp. 1099-1112, 2008.

[12] A. Negesse, T. Getaneh, H. Temesgen, T. Taddege, D. Jara, and Z. Abebaw, "Prevalence of anemia and its associated factors in human immuno deficiency virus infected adult individuals in Ethiopia. A systematic review and meta-analysis," BMC Hematology, vol. 18, no. 32, pp. 1-10, 2018.

[13] N. E. Assefa, H. Berhe, F. Girma et al., "Risk factors of premature rupture of membranes in public hospitals at Mekele city, Tigray, a case control study," BMC Pregnancy and Childbirth, vol. 18, no. 1, p. 386, 2018.

[14] M. Assefa, W. E. Abegaz, A. Shewamare, G. Medhin, and M. Belay, "Prevalence and correlates of anemia among HIV infected patients on highly active anti-retroviral therapy at Zewditu Memorial Hospital, Ethiopia," BMC Hematology, vol. 15, no. 1, p. 6, 2015.

[15] K. W. Zerihun, G. A. Bikis, and E. A. Muhammad, "Prevalence and associated factors of anemia among adult human immune deficiency virus positive patients on anti-retroviral therapy at Debre Tabor Hospital, Northwest Ethiopia," BMC Research Notes, vol. 12, no. 1, p. 168, 2019.

[16] National AIDS Resource Center, HIV/AIDS Estimates and Projections in Ethiopia, National AIDS Resource Center, Addis Ababa, Ethiopia, 2011, http://fitun.etharc.org/ resources/finish/53-hiv-aids-estimates-and-projections/327hiv-aids-estimates-and-projections-in-ethiopia-2011-2016.

[17] T. Yohannes, T. Laelago, M. Ayele, and T. Tamrat, "Mortality and morbidity trends and predictors of mortality in underfive children with severe acute malnutrition in Hadiya zone, South Ethiopia: a four-year retrospective review of hospitalbased records (2012-2015)," BMC Nutrition, vol. 3, no. 1, p. 18, 2017.

[18] A. G. Alamdo, T. Fiseha, A. Tesfay, M. K. Deber, Z. M. Tirfe, and T. Tilahun, "Anemia and its associated risk factors at the time of antiretroviral therapy initiation in public health facilities of Arba Minch town, southern Ethiopia," Health, vol. 7, no. 12, pp. 1657-1664, 2015.

[19] A. Tsegaye, T. Messele, T. Tilahun et al., "Immunohematological reference ranges for adult Ethiopians," Clinical and Diagnostic Laboratory Immunology, vol. 6, no. 3, pp. 410-414, 1999.

[20] E. Munyazesa, I. Emile, E. Mutimura et al., "Assessment of haematological parameters in HIV-infected and uninfected Rwandan women: a cross-sectional study," BMJ Open, vol. 2, no. 6, Article ID e001600, 2012.

[21] S. Takuva, M. Maskew, A. T. Brennan, I. Sanne, A. P. MacPhail, and M. P. Fox, "Anemia among HIV-infected patients initiating antiretroviral therapy in South Africa: improvement in hemoglobin regardless of degree of immunosuppression and the initiating ART regimen," Journal of Tropical Medicine, vol. 2013, Article ID 162950, 6 pages, 2013.

[22] R. Omoregie, E. Omokaro, O. Palmer et al., "Prevalence of anaemia among HIV-infected patients in Benin City, 
Nigeria," Tanzania Journal of Health Research, vol. 11, no. 1, pp. 1-4, 2009.

[23] P. Petraro, E. Hertzmark, D. Sando et al., "Determinants of anemia among human immunodeficiency virus-positive adults at care and treatment clinics in dar es salaam, Tanzania," The American Journal of Tropical Medicine and Hygiene, vol. 94, no. 2, pp. 384-392, 2016.

[24] E. J. Santiago-Rodríguez, A. M. Mayor, D. M. FernándezSantos, Y. Ruiz-Candelaria, and R. F. Hunter-Mellado, "Anemia in a cohort of HIV-infected Hispanics: prevalence, associated factors and impact on one-year mortality," BMC Research Notes, vol. 7, no. 1, p. 439, 2014.

[25] C. Martin, K. Poudel-Tandukar, and K. C. Poudel, "HIV symptom burden and anemia among HIV-positive individuals: cross-sectional results of a community-based positive living with HIV (POLH) study in Nepal," PLoS One, vol. 9, no. 12, Article ID e116263, 2014.

[26] J. A. Tiewsoh, B. Antony, and R. Boloor, "Seroprevalence of HIV-2 and dual infection among HIV-infected individuals with clinical and laboratory features at a tertiary care teaching Hospital, Mangalore: the present scenario," Annals of African Medicine, vol. 18, no. 2, pp. 70-74, 2019.

[27] C. Obirikorang, R. G. Issahaku, D. N. M. Osakunor, and J. Osei-Yeboah, "Anaemia and iron homeostasis in a cohort of HIV-infected patients: a cross-sectional study in Ghana," AIDS Research and Treatment, vol. 2016, Article ID 1623094, 8 pages, 2016.

[28] K. B. Gebremedhin and T. B. Haye, "Factors associated with anemia among people living with HIV/AIDS taking ART in Ethiopia," Advances in Hematology, vol. 2019, Article ID 9614205, 8 pages, 2019.

[29] F. Masaisa, J. Mukiibi, J. B. Gahutu, J. Philippé, and J. Delanghe, "Anemia in human immunodeficiency virusinfected and uninfected women in Rwanda," The American Journal of Tropical Medicine and Hygiene, vol. 84, no. 3, pp. 456-460, 2011.

[30] H. Melese, M. M. Wassie, H. Woldie, A. Tadesse, and N. Mesfin, "Anemia among adult HIV patients in Ethiopia: a hospital-based cross-sectional study," HIV/AIDS-Research and Palliative Care, vol. 9, pp. 25-30, 2017. 